\title{
Cancer treatment: changes in hearing thresholds
}

\author{
Larissa Azevedo Ramalho Machado², Tarsila Santos Amaral ${ }^{2}$, Lisley Kelly Santos de Aguiar $^{2}$, Aline Cabral de Oliveira ${ }^{3}$ and Priscila Feliciano \\ de Oliveira ${ }^{1 *}$ \\ ${ }^{1} \mathrm{PhD}$ in Health Sciences, Universidade Federal de Sergipe (UFS), São Cristóvão, SE, Brazil \\ ${ }^{2}$ Speech and Audiologist Terapist, Universidade Federal de Sergipe (UFS), São Cristóvão, SE, Brazil \\ ${ }^{3}$ Postdoctoral in Speech Pathology, Universidade Federal de São Paulo (UNIFESP), São Paulo, SP, Brazil
}

\begin{abstract}
Purpose: Evaluate changes in hearing thresholds in patients undergoing cancer treatment.

Method: Prospective, longitudinal study carried out at Cancer Center of a Sergipe's public hospital, Brazil. Audiological evaluation (Conventional Pure Tone Audiometry) have been performed in 20 patients following eight weeks of cancer treatment (reference exam) and 20 weeks (sequential exam) of treatment. All survivors had normal hearing thresholds before cancer treatment. The procedure has been approved by the Ethics in Research Committee, under protocol \# 33665014.7 .0000 .5546 .

Results: Head and neck cancer displayed oncology highest incidence (35\%). Referential exam, eight weeks before treatment, displayed 15\% with sensorineural hearing loss and $17.5 \%$ at second tests. It was observed a slight worsening for all test frequencies of referential and sequential exam. Tinnitus was the biggest complaint $(81.3 \%)$, but there was no statistic correlation between hearing loss $(\mathrm{p}=0.89)$.

Conclusion: Hearing monitoring form this small sample suggest chemotherapy and radiotherapy treatments can have a detrimental effect on hearing thresholds significate for 2 to $8 \mathrm{kHz}$ eight weeks after cancer treatment and 4 to $8 \mathrm{kHz} 20$ weeks before.
\end{abstract}

\section{Introduction}

Since 1960, cancer has been the major cause of death in the world. The estimation of this desease in Brazil for 32019 is 600 thousand of new cases. In Aracaju (Brazilian north east), numbers have been increasing significantly, once INCA notified 3.610 cases in 2010 and in 2014, 4.755 cases [1]. For 2018/2019 there will be an estimation of 4.930 new cases in Aracaju, Sergipe.

Treatment methods have been widely used. There is an increase of successful outcomes and the cure of some cancers is well documented. Despite it, chemotherapy and radiotherapy lead to deleterious collateral effects. One of them is hearing loss, which can affect middle or inner ear [2]. Generally, hearing loss is sensorineural, bilateral and irreversible, tends to be permanent and it has tinnitus as a symptom [3,4]. It is a result of ototoxicity, which can damage cochlea's basal turn and hits outer hair cells, before some days or weeks after cancer treatment [5].

It is essential to follow-up patient's hearing system. Hearing loss affects either communication, social and emotional aspects; it may furhter reduce quality of life. Moreover, investigators have described hearing loss during cancer treatment, which can became worse (75\% up to a $100 \%$ ) especially in higher frequencies $[3,5]$. Audiological procedure, as conventional pure tone audiometry, is the basic of audiological battery for hearing loss diagnostic, once ototoxicity can develop a high frequency damage [6].

Early diagnosis and hearing assessment is essential to identify the hearing loss early. It reduces impact on the individual's life by means of proper medical and hearing intervention [7]. However, it is difficult to monitor hearing thresholds, once most of patients are debilitated and die before finishing treatment, that's why there are few studies with longitudinal audiological follow-up [8].

The aim is to evaluate changes in hearing thresholds of patients undergoing cancer treatment.

\section{Method}

Prospective, longitudinal, transversal cohort study has been carried out at a Public Hospital in Aracaju, Brazil. The Ethics and Research Committee approved it under protocol \#33665014.7.0000.5546. All patients agreed to undergo hearing test, and all provided written informed consent for participation in this study.

It was recorded serial audiometric evaluations of 20 cancer treatment's patients in two years (2015/2016). All patients came from free demand or by medical referral. To be part of the research, all participants should have medical cancer diagnosis, be on chemotherapy and/or radiotherapy treatment and have a normal hearing threshold before the cancer treatment.

Patients who had ear surgery, history of acoustic noise-induced hearing loss trauma, ear infections, congenital syndromes and obstruction of the external acoustic meatus and those who previously had received

*Correspondence to: Priscila Feliciano de Oliveira, Departamento de Fonoaudiologia, Universidade Federal de Sergipe. Av. Marechal Rondon, s/n. São Cristóvão, se, Brazil, Cep: 49100-000, Tel: +55 79 3194-6805/+55 79 3194-6842; E-mail: oliveirapriscila@hotmail.com

Key words: cancer, chemotherapy, hearing loss, radiotherapy

Received: March 26, 2018; Accepted: April 17, 2018; Published: April 24, 2018 
chemotherapy and/or radiotherapy treatment were excluded from the study.

As a procedure, anamnesis and conventional pure tone audiometry were part of the evaluation.

Researchers have developed an anamnesis to collect patient's medical history as previous history of cancer (age at diagnosis, type and extent of disease, type of treatment, symptom) and hearing aspects (hearing complaints and previous otological history).

Hearing threshold (air conduction) were recorded across all octave frequencies from 250 to $8.000 \mathrm{~Hz}$. Bone conduction test was recorded for all patients between 0.5 to $4 \mathrm{kHz}$ frequencies. A hearing threshold of $26 \mathrm{~dB} \mathrm{HL}$ was considered significant for hearing impairment, so hearing threshold $0-25 \mathrm{~dB}$ HL was defined as normal. Mild, moderate, severe and profound hearing loss were defined as hearing thresholds in the range of $26-40 \mathrm{~dB} \mathrm{HL}, 41-70 \mathrm{~dB} \mathrm{HL}, 71-90 \mathrm{~dB} \mathrm{HL}$ and +91dB HL, respectively [9]. Audiology records were taken with a pure tone-screening audiometer Ad28 (Interacoustics, Denmark) with TDH 39 earphones.

Two audiograms were acquired during the treatment: referential (first exam) was collected eight weeks before the cancer treatment and sequential exam (second exam), 20 weeks before. During the treatment, all patients were receiving chemotherapy and radiotherapy. Referential exams were compared with sequential exam based in American SpeechLanguage-Hearing Association criteria (ASHA, 1994). Comparing the two exams, it was considered normal or acceptable variability in hearing threshold a difference of $10 \mathrm{~dB}$ HL in a single frequency; variability more than $20 \mathrm{~dB}$ HL in a single frequency or $10 \mathrm{~dB}$ HL in two or more frequencies was considered thresholds increase [10].

The data analysis for this study was generated using Statistical Package for Social Sciences 20 version (SPSS, Chicago, USA). Normality of distribution was assessed using the Kolmogorov Test. Relationships of continuous variables with the presence/absence of hearing loss were valuated with the Mann Whitney Test, while categorical data were compared using the Chi-squared test. $\mathrm{P}$ values of less than 0.05 were considered statistically significant.

\section{Results}

The present study of audiological assessment set out 20 cancer patients, whose avarage age were $33.5( \pm 15,66)$ years, $55.5 \%$ were female, $35 \%$ had head and neck cancer and $15 \%$ breast cancer.

Tinnitus was present in $81.3 \%$ of all patients with no significant difference between those who had hearing loss and normal hearing thresholds ( $\mathrm{p}=0.11$; Chi-square Test). During the treatment, at sequential exam, $20 \%$ reported that tinnitus gets worse.

Audiological findings displayed 15\% with sensorioneural hearing loss as from $3 \mathrm{kHz}$ frequency at referential exam. $28.8 \%$ of head and neck cancer's patients were diagnosed with hearing loss. Data analysis revealed a statistical significant difference between those who had hearing loss and normal hearing thresholds from 2 up to $8 \mathrm{kHz}$ in referential exam (Figure 1).

Figure 2 describes sequential audiological findings. It was diagnosed $17.5 \%$ with sensorioneural hearing loss with statistical significant difference between hearing loss and normal hearing thresholds from 4 up to $8 \mathrm{kHz}$.

The comparison of referential and sequential exams by ASHA (1994) criteria demonstrated that 35\% of audiograms had increased hearing thresholds. One patient had a normal hearing threshold in referential exam, but the sequential exam displayed hearing loss.
Moreover, normal hearing thresholds had a statistical significant difference of $4 \mathrm{kHz}$ between referential and sequential exam (Figure 3).

On the other hand, patients who was diagnosed with hearing loss in referential exam had equal audiological findings in sequential exam, except for $0.25 \mathrm{kHz}$. There was a statistical significant difference of 0.25 $\mathrm{kHz}$ between referential and sequential exam (Figure 4).

\section{Discussion}

The incidence of cancer type was 35\% for head and neck in male. Head and neck cancer occupies the fifth position of all men's cancer. There is association of this type of cancer with tobacco and alcohol

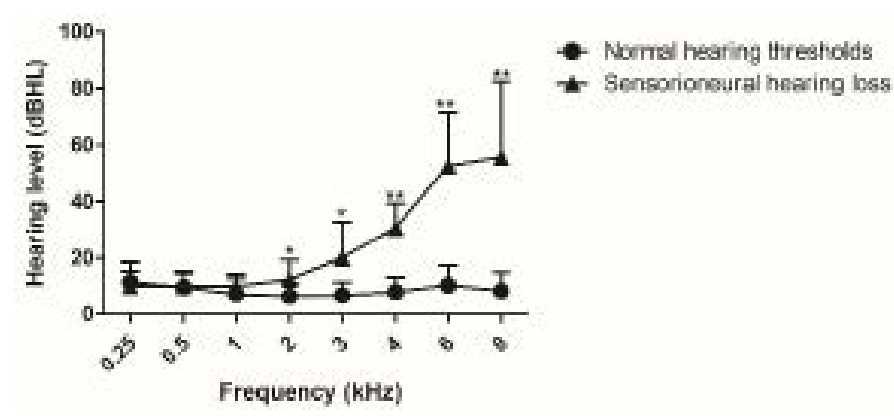

Figure 1. Referential hearing thresholds, as measured by conventional pure tone audiometry evaluated five weeks after cancer treatment. Normal hearing threshold $n=34$ and sensorioneural hearing threshold $\mathrm{n}=06$. For analysis, Mann Whitney test was used. Values are expressed as mean $\pm \mathrm{SD} .\left({ }^{*}\right) p<0.05$ and $\left({ }^{* *}\right) p<0.01$ vs. normal hearing threshold. dB HL: decibel hearing level

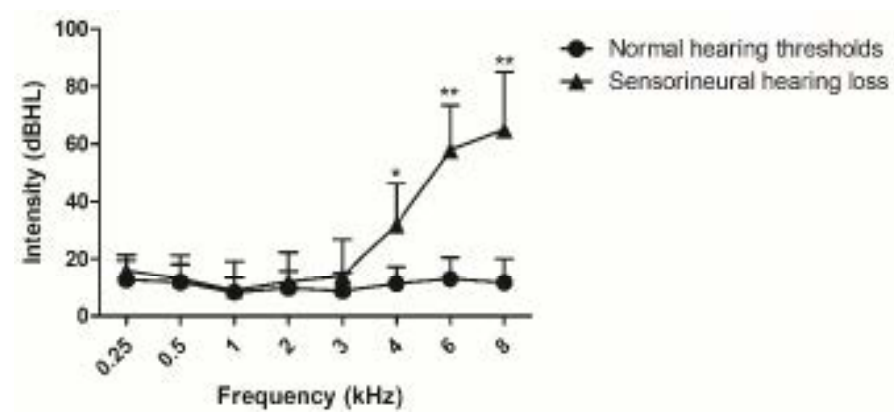

Figure 2. Sequential hearing thresholds, as measured by conventional pure tone audiometry evaluated ten weeks after cancer treatment. Normal hearing threshold $n=33$ and sensorioneural hearing threshold $\mathrm{n}=07$. For analysis, Mann Whitney test was used. Values are expressed as mean $\pm \mathrm{SD}$. $(*) p<0.05$ and $(* *) p<0.01$ vs. normal hearing threshold. dB HL: decibel hearing level

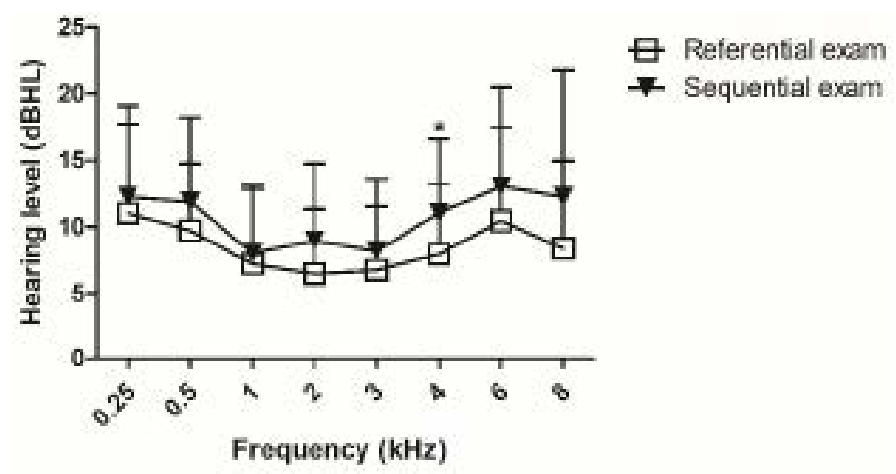

Figure 3. Normal hearing thresholds of referential and sequential exams, as measured by conventional pure tone audiometry. Referential exam $n=34$ and sequential exam $n=33$. For analysis, Mann Whitney test was used. Values are expressed as mean $\pm \mathrm{SD} .\left(^{*}\right) p<0.05 \mathrm{vs}$. referential exam. dB HL: decibel hearing level 


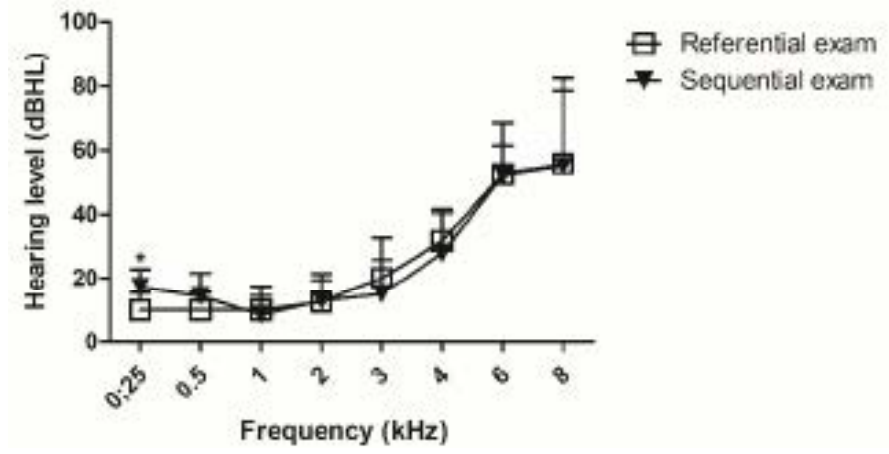

Figure 4. Hearing loss thresholds of referential and sequential exams, as measured by conventional pure tone audiometry. Referential exam $n=06$ and sequential exam $n=07$. For analysis, Mann Whitney test was used. Values are expressed as mean \pm SD. $(*) p<0.05$ vs referential exam. dB HL: decibel hearing level.

consumption [11]. On the other side, in our study, women were diagnosed with breast cancer (15\%). Studies reported high incidence of breast cancer in women and it is the fourth most common oncologic diagnose in adults $[11,12]$.

Hearing loss was diagnosed in $15 \%$ of all cancer patients. There is a variability of hearing loss incidence, once it has influence of radiation technique (2D or 3D) and dose, anticancer drugs and dose, individual susceptibility and age $[3,13]$. In our research, all patients received, and $3 \mathrm{D}$ radiotherapy as prescribed by oncologist. As chemotherapeutic drugs were carboplatin, cyclophosphamide and doxorubicin, which can damage permanently organ of hearing as described $[3,14,15]$. Investigators described that platinnun drugs affected $14 \%$ of cancer treatment's survivors once these drugs are considered not only ototoxic, but neurotoxic [15]. The incidence of hearing loss for head and neck cancer, in our study was $28.8 \%$ therefore investigators reported an incidence of $27 \%$ to $95 \%$ [13].

Our audiometric configuration results were characterized by sensorioneural hearing loss. Indeed, investigators reported that most of hearing loss in oncology survivors is sensorineural [5,16-18], which affects inner ear, particularly outer hair cells $[3,17]$. Inner ear is the most susceptible organ in a durable long-term radiotherapy and chemotherapy. Cancer treatment leads to an inner ear vascular insufficiency, reduces number of capillaries, degenerates endothelial vessels, loss of cells of Organ of Corti, degenerates striavascularis and leads to spiral ganglion cells and cochlear nerve atrophy $[6,19]$.

Radiotherapy is the most common treatment for head and neck cancer, initially patients can have acute middle ear side effects; therefore, they can develop an inner ear hearing loss. As described, chemotherapy uses drugs like Cisplatin, Carboplatin, Oxaliplatin, and Nedaplatin, which have been widely chosen as an efficient treatment in oncology. Moreover, platinnun group is the most devastating drug to inner ear $[3,14,15]$. It has toxic side effects to the peripheral auditory system which lead to hearing loss with an impaired social communication [3].

A large number of patients, in our research complained about tinnitus $(81.3 \%)$. Tinnitus is a common condition, especially in cancer treatment, caused by chemotherapy drugs and radiotherapy $[17,20$ 22]. The present study did not reveal relation between tinnitus and hearing loss, in contrast investigators have reported this relation [20]. Chemotherapy with platinnun and / or taxane compounds develops neurotoxicity, which induces neuropathy. Investigators reported hearing loss, balance problems and tinnitus with significant decrement of quality of life [15].
The incidence of hearing loss was observed at frequencies above $3 \mathrm{KHz}$, in our study. Indeed, investigators reported hearing loss at high frequencies $[5,8,23]$. Only radiotherapy as head and neck cancer treatment reported hearing loss at frequencies above $4 \mathrm{kHz}$ [13].

In our study, follow-up exams showed that $15 \%$ had sensorineural hearing loss in referential exam and it increased to $17.5 \%$ (sequential exam). One study reported a $75 \%$ of hearing thresholds worsening [7]. Most of studies evidenced that hearing thresholds during cancer treatment get worse $[5,7]$.

\section{Conclusion}

The results from this small sample suggest that chemotherapy and radiotherapy treatments can have a detrimental effect on hearing thresholds from 4 to $8 \mathrm{kHz}$ eight weeks before cancer treatment and 2 to $8 \mathrm{kHz} 20$ weeks after.

\section{Authorship and contributions}

Machado, L.A.R, Amaral, T.S, Aguiar, L.K.S participated in audiometric evaluation procedure. Oliveira, A.C participated in the statistical analyses and discussion of results. Oliveira, P.F. conducted the research and participated in all stages of manuscript preparation.

\section{Acknowledgments}

This paper would not have been possible without the patient's participation and contributions of all people of Sergipe's Cancer Care Center that have been working on it every day.

\section{Declaration of interest}

The authors report no conflicts of interest.

\section{References}

1. Instituto Nacional de Câncer. Tratamento do câncer. Available from: http://www2.inca gov.br/wps/wcm/connect/cancer/site/tratamento

2. Malgonde MS, Nagpure PS, Kumar M (2014) Audiometric patterns in ototoxicity after radiotherapy and chemotherapy in patients of head and neck cancers. Indian J Palliat Care 21: 164-167. [Crossref]

3. Paken J, Govender CD, Pillay M, Sewram V (2016) Cisplatin-Associated Ototoxicity: A Review for the Health Professional. J Toxicol. [Crossref]

4. Goel H, Laad P, Naik A (2013) The study of auditory effects after concomitant radiotherapy and chemotherapy in patients with head and neck cancer. Indian J Otol 19: 136-139.

5. Dell'Aringa AHB, Isaac ML, Arruda GV, Dell'Aringa AR, Esteves MC (2010) Audiological findings in pacients treated with radiotherapy for head and neck tumors. Braz J Otorhinolaryngol 76: 527-532. [Crossref]

6. Magalhães F, Ribeiro D, Tomé D, Quelhas H, Lobão M, et al. (2012) Effects on Hearing in Patients with Neoplasm of Head and Neck and Brain Tumours undergoing Radiotherapy and Combined Therapy. Arq Med 26: 141-144.

7. Mota LAA, Melo MSI, Santos MHP, Albuquerque KMG de, Tavares C de L (2007) Ototoxidade da cisplatina: série de casos. Rev Assoc Med Bras 53: 370-373.

8. Garcia AP, Iório MCM, Petrilli AS (2003) Audiological monitoring of cisplatin exposed patients. Rev Bras Otorrinolaringol 69: 215-221.

9. Silman S, Silverman C (1997) Basic Audiology Testing. Auditory Diagnosis: Principles and Applications. SingularPublishing Group : 10-65.

10. Durrant JD, Campbell K, Jacobson G, Lonsbury-Martin BL (2009) American Academy of Audiology Position Statement and Clinical Practice Guidelines: Ototoxicity Monitoring Available from:https://audiologyweb.s3.amazonaws.com/migrated/ OtoMonGuidelines.pdf_539974c40999c1.58842217.pdf

11. Instituto Nacional de Câncer (2017) Estimativa 2018: incidência de câncer no Brasil INCA, editor. Rio de Janeiro: Instituto Nacional de Câncer José Alencar Gomes da Silva. Coordenação de Prevenção e Vigilância. 
12. Instituto Nacional de Câncer José Alencar Gomes da Silva. Ministério da saúde (2013) Estimativas 2014: Incidência de câncer no Brasil. http://www2.inca.gov.br/wps/wcm/ connect/agencianoticias/.

13. Mujica-Mota M, Waissbluth S, Daniel SJ (2013) Characteristics of radiation-induced sensorineural hearing loss in head and neck cancer: A systematic review. Head Neck 35: 1662-1668. [Crossref]

14. Sheth S, Mukherjea D, Rybak LP, Ramkumar V (2017) Mechanisms of CisplatinInduced Ototoxicity and Otoprotection. Front Cell Neurosci 11: 338. [Crossref]

15. Miaskowski C, Mastick J, Paul SM, Abrams G, Cheung S, et al. (2018) Impact of chemotherapy-induced neurotoxicities on adult cancer survivors' symptom burden and quality of life. J Cancer Surviv 12: 234-245. [Crossref]

16. Hudson MM, Ness KK, Gurney JG, Mulrooney DA, Chamaitilly W, et al. (2013) Clinical ascertainment of health outcomes among adults treated for childhood cancer. JAMA 309: 2371-2381. [Crossref]
17. Landier W (2016) Ototoxicity and cancer therapy. Cancer 122: 1647-1658. [Crossref]

18. Schultz C, Goffi-Gomez MVS, Liberman PHP, Carvalho AL (2009) Report on hearing loss in oncology. Braz J Otorhinolaryngol 75: 634-641. [Crossref]

19. Kırca K, Kutlutürkan S (2017) Symptoms of patients with head and neck cancers undergoing radiotherapy. Eur J Cancer Care 26. [Crossref]

20. Kim H-J, Lee H-J, An S-Y, Sim S, Park B, et al. (2015) Analysis of the prevalence and associated risk factors of tinnitus in adults. PLoS One 10: e0127578. [Crossref]

21. Arora R, Thakur JS, Azad RK, Mohindroo NK, Sharma DR, et al. (2009) Cisplatinbased chemotherapy: Add high-frequency audiometry in the regimen. Indian J Cancer 46: 311-317. [Crossref]

22. Gilligan T (2015) Quality of life among testis cancer survivors. Urol Oncol 33: 413 419. [Crossref]

23. Bhandare N, Jackson A, Eisbruch A, Pan CC, Flickinger JC, et al. (2010) Radiation therapy and hearing loss. Int J Radiat Oncol Biol Phys 76: S50-57. [Crossref]

Copyright: (C2018 Machado LAR. This is an open-access article distributed under the terms of the Creative Commons Attribution License, which permits unrestricted use, distribution, and reproduction in any medium, provided the original author and source are credited. 